\title{
Clinical impact of the immunochemical fecal occult blood test for colorectal cancer screening in Brazil
}

\author{
Cláudio R. Teixeiraa , Michele L. Bonotto ${ }^{\mathrm{a}, \mathrm{b}}$, Júlio P. Lima ${ }^{\mathrm{a}, \mathrm{b}}$, Luciana F. Figueiredo ${ }^{\mathrm{a}, \mathrm{b}}$, Leonardo Conrado ${ }^{\mathrm{a}, \mathrm{b}}$, \\ Carlos Frasca ${ }^{a, b}$
}

Moinhos de Vento Hospital; Fugast, Rio Grande do Sul, Brazil

Abstract

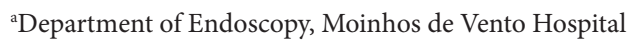

(Cláudio R. Teixeira, Michele L. Bonotto, Júlio P. Lima,

Luciana F. Figueiredo, Leonardo Conrado, Carlos Frasca);

${ }^{\mathrm{b}}$ Gastroenterology Fundation of Rio Grande do Sul, Fugast, Endoscopy

Unit (Michele L. Bonotto, Júlio P. Lima, Luciana F. Figueiredo,

Leonardo Conrado, Carlos Frasca), Brazil

Conflict of Interest: Claudio Rolim Teixeira is a speaker/consultant of Fujifilm Co.

Correspondence to: Cláudio Rolim Teixeira, MD, PhD, Colonoscopy Center, Quintino Bocaiuva, 683 conj 502, 90440151 Porto Alegre, Rio Grande do Sul, Brazil, Tel./Fax: +55 5133 310656,

e-mail: centrodecolonoscopia@gmail.com

The research was carried out at Gastroenterology Foundation of Rio Grande do Sul, Endoscopy Unit. The current institution of the corresponding author is Moinhos de Vento Hospital

Received 12 December 2017; accepted 3 March 2017; published online 27 April 2017

DOI: https://doi.org/10.20524/aog.2017.0151

\section{Introduction}

CRC is the third most common neoplasia in the world and the fifth cause of cancer-related death in Southern Brazil [1]. There has been a significant increase in the global CRC incidence over the last 20 years and a still greater increase in CRC incidence is expected by 2025, due to population aging [2]. CRC screening by colonoscopy with detection and removal of pre-malignant lesions or early cancers is the gold standard for reducing cancer-related mortality and morbidity [3].

CRC screening by occult blood testing is also reported to reduce cancer-related mortality [4]. Occult blood testing has advantages in population screening programs in terms of patient's acceptance, availability, and cost-effectiveness, and because it is a risk-free procedure [4]. However, both negative and positive results require confirmatory colonoscopy for a definitive diagnosis.

The guaiac fecal occult blood test (g-FOBT) is a widely used test for detecting fecal occult blood. However, this test 
has many drawbacks, such as the need for a diet in the days prior to the test, avoidance of some medications, and the need to avoid dental brush procedures up to three days before beginning the fecal collection for the test. Furthermore, it has a low sensitivity, specificity and positive predictive value [5]. In the last few years, the immunochemical fecal occult blood test (i-FOBT) has been demonstrated to have better reproducibility, as well as higher sensitivity, specificity and positive predictive value than the g-FOBT [6]. In addition, there is no need for a special diet prior to fecal testing or other special precautions [7]. Consequently, this method has found better acceptance among patients and physicians [5]. The OC-Light immunochemical fecal occult blood test (i-FOBT, EIKEN chemical Co., Tokyo, Japan) is a qualitative test that can detect $50 \mathrm{ng} / \mathrm{mL}$ of hemoglobin $(\mathrm{Hb})[2,3]$; furthermore, it is specific for human $\mathrm{Hb}$, while upper digestive bleeding does not produce a positive test. The low level of detection of OC-Light provides high sensitivity, which is fundamental for improving the detection rate of CRC in population screening programs.

The aim of this study was to evaluate the feasibility, sensitivity, and specificity of the i-FOBT for colonic neoplasms in an average-risk population in Southern Brazil, considering colonoscopy as the diagnostic gold standard.

\section{Patients and methods}

A population-based study was designed in which averagerisk volunteers who where symptom-free for colonic disease were enrolled between April 2015 and January 2016 in two gastroenterology referral centers (Gastroenterology Foundation of Rio Grande do Sul, Fugast, Endoscopy Unit, and Santa Casa Hospital). A total of 1039 volunteers agreed to participate in the study. All volunteers agreed to take the test home and carry it out, but in case of non compliance the participants were contacted by phone. Only 321 (30.9\%) participants returned to undergo colonoscopy.

The tests were analyzed in a laboratory in one of the Gastroenterology Units participating in the study (Gastroenterology Foundation of Rio Grande do Sul, Fugast, Endoscopy Unit). The samples were analyzed using the OC-Light ${ }^{\text {Tx }}$ by two independent investigators; $\mathrm{Hb}$ was detected and results were given automatically as $10 \mu \mathrm{g} \mathrm{Hb} / \mathrm{g}$ feces The OC-Light i-FOBT is an immunoassay that uses a blend of a polyclonal and monoclonal antibodies to specifically detect the presence of $\mathrm{Hb}$ in feces. The sample end of the test strip is dipped in the feces extract. The liquid feces wicks through a series of absorbent materials and contacts latex particles conjugated to an antibody specific to $\mathrm{Hb}$. The sample and latex conjugate then wick through a membrane that contains zones of immobilized antibodies, a patient test zone of anti-Hb capture antibody and a control zone of anti-mouse antibody. If $\mathrm{Hb}$ is present in the sample it serves to link the latex conjugate to the capture antibody in the patient test zone. The control zone antibody binds the monoclonal antibody on the latex. The buildup of latex particles in the zones leads to the development of visible blue bands. The cutoff for a positive test is $10 \mu \mathrm{g} \mathrm{Hb} / \mathrm{g}$ feces $(50 \mathrm{ng} / \mathrm{mL}$ ).
Colonoscopy screening was offered to all volunteers, regardless of whether they were positive or negative for i-FOBT. Lesion size and location were registered. The resected lesions were sent for histopathological analysis. Advanced CRCs, for which endoscopic resection was not feasible, were biopsied. All other colonic diseases were also evaluated and registered. Those performing the colonoscopy and histologic examinations were blinded to the i-FOBT results. The Ethics Committee of the Federal University of Health Science approved the study under number 41858515.8.0000.5345.

Statistical analysis was performed using SPSS software for Windows, version 18.0. Formal descriptive statistics for proportions and continuous variables were calculated with their respective confidence interval $(95 \% \mathrm{CI})$. The acceptance and return percentage were registered. Once the colonoscopy was performed in positive and negative participants for i-FOBT, the sensitivity and specificity of the test could be estimated directly.

\section{Results}

Overall, 1039 participants (675 female), with a mean age 60.5 years (range 50-75) were included in the study. Nine-hundred forty-eight (91.2\%) returned with the i-FOBT (95\%CI 89.4-92.9). Ninety-two (9.7\%) were positive (95\%CI 7.9-11.8) and of these 73 (79.3\%) underwent colonoscopy. The remaining 19 (20.7\%) participants refused to undergo a colonoscopy (Fig. 1). The cecal intubation rate was $100 \%$.

Nine $(12.3 \%)$ advanced CRCs were detected among the 73 participants with a positive i-FOBT. Two patients $(2.7 \%)$ had early CRC, 7 patients (9.5\%) had high-grade dysplasia adenomas and $25(34.2 \%)$ patients had low-grade dysplasia adenomas. Thirty $(41 \%)$ cases had a normal colon or no neoplastic lesions (Fig. 2). Of the 243 negative i-FOBT cases who underwent colonoscopy, one $(0.4 \%)$ had advanced CRC and 91 patients $(37.6 \%)$ had low-grade dysplasia adenomas. The remaining 151 (62\%) had normal colonoscopy or no neoplastic lesions (Fig. 3).

The detection rate of CRC considering the whole screened population ( $\mathrm{n}=1039)$ was $1.05 \%(11 / 1039)$, and the detection rate for the population who underwent colonoscopy was $3.48 \%$ $(11 / 316)$. High-grade dysplasia adenomas were detected in $0.67 \%(7 / 1039)$ of the screened population and $2.2 \%(7 / 316)$ of those screened by colonoscopy. The participation rate was $91.2 \%$ (948/1039). The positivity rate of the method was $9.7 \%$. Table 1 shows the results of i-FOBT OC-Light analysis for the detection of CRC, adenoma and any neoplastic lesion in a Southern Brazilian population.

\section{Discussion}

This is the first CRC screening study with OC-Light (i-FOBT) to be performed in a Brazilian population. i-FOBT screening programs have shown a significant reduction in CRC mortality 


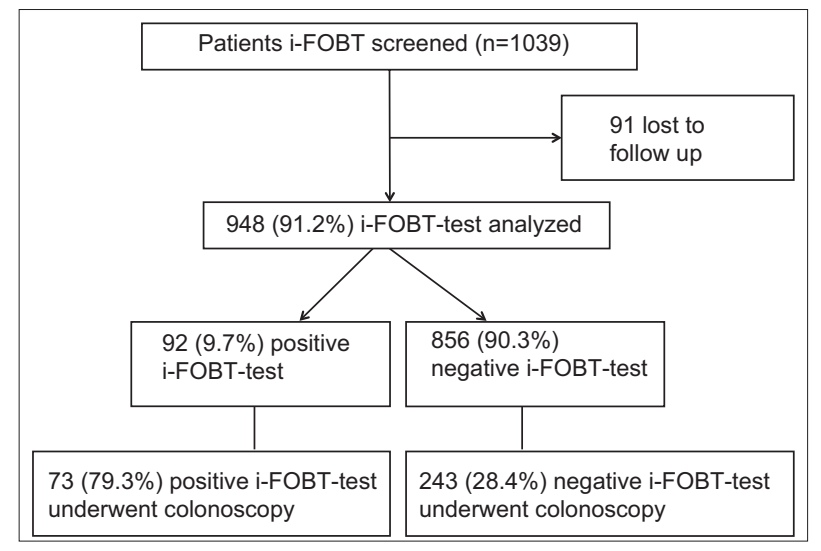

Figure 1 Flow chart of the study i-FOBT, immunochemical fecal occult blood test

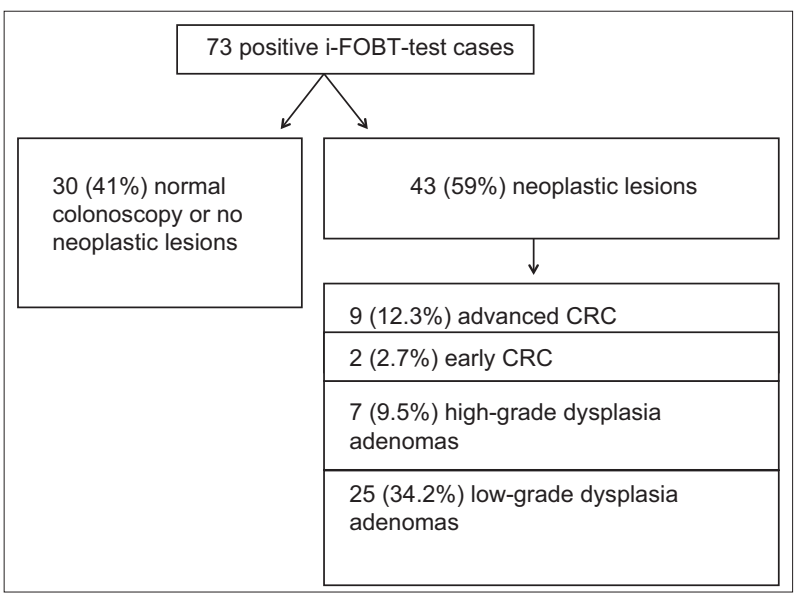

Figure 2 Colonoscopy results of participants with a positive immunochemical fecal occult blood test (i-FOBT) CRC, colorectal cancer

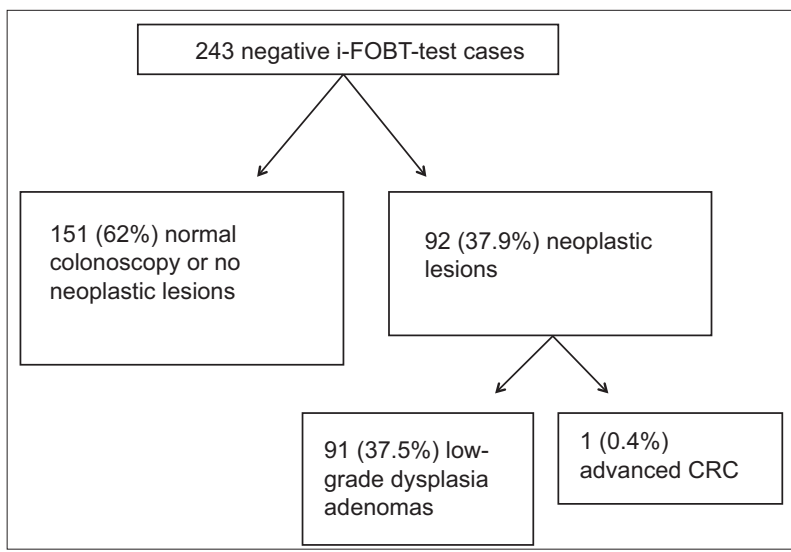

Figure 3 Colonoscopy results of participants with a negative immunochemical fecal occult blood test (i-FOBT)

and their impact on incidence rates has already been described in the literature $[10,11]$. The reduction in incidence rates associated with the removal of precancerous lesions may reflect an even greater reduction in mortality in the long term [9].
The applicability of this test to CRC screening was excellent, with a high acceptance rate among our patient population. Similar figures were observed in another South American CRC screening study, in which $90 \%$ of the participants returned with stool samples [4]. The high acceptance of this test supports our ongoing screening program. We believe that the absence of dietary restrictions is paramount for the acceptability of this test within our population, where people consume large amounts of meat.

The positivity rate of our study resembles that of other published results $[4,5]$. The most striking results of our study were the high sensitivity for CRC detection and the high negative predictive value of the test. However, the negative predictive value for harboring any neoplastic lesion was low. This high sensitivity demonstrates that the i-FOBT is a promising test for CRC screening in the general population older than 50 years. The OC-Light i-FOBT test is designed for preliminary screening.

Chemical tests, such as the guaiac method, have been reported to reduce CRC mortality in a cost-effective fashion. However, this method had a low specificity because the $\mathrm{Hb}$ detected is pseudoperoxidase activity $\mathrm{Hb}$, not specific to human $\mathrm{Hb}$. This gives this test a high false positive rate, besides the need for dietary restrictions and discontinuation of drugs and teeth brushing $[8,9]$.

We found a relatively high adenoma detection rate, but our figures for CRC and high-grade dysplasia adenomas were in line with other published results $[10,11]$. Our study had the limitation that only a relatively small number of volunteers were evaluated by colonoscopy. However, we also evaluated those participants with a negative test, although in a smaller percentage than those with a positive-test. This allowed a more accurate evaluation in relation to sensitivity and specificity. Given that we offered colonoscopy to all volunteers, regardless of their i-FOBT result, the low rate of acceptance demonstrates that many volunteers are reluctant to undergo an invasive procedure. Thus, i-FOBT can also be a useful tool to reinforce the need for colonoscopy, mainly in those volunteers who are positive; this could increase the chance of CRC detection, expanding screening programs and hence reducing the incidence of CRC. On the other hand, the low acceptance of colonoscopy could make it difficult to analyze the sensitivity and specificity of the test.

Onelimitation of the method is that i-FOBT could be affected by certain medications, such as aspirin and non-steroidal antiinflammatory drugs, because they may cause gastrointestinal erosions and subsequent bleeding in some patients, leading to positive results. However, a recent publication demonstrated that the positive predictive value of i-FOBT was not affected by ongoing antithrombotic therapy [12]. Another advantage of $\mathrm{i}-\mathrm{FOBT}$ is that a positive test may be useful for predicting small bowel disease in patients with occult obscure gastrointestinal bleeding and negative colonoscopy [9].

Our findings confirm that i-FOBT is specific for predicting lesions in the lower gastrointestinal tract. The sensitivity for CRC detection is more than $90 \%$. The acceptance rate was excellent, with $91.2 \%$ returning the i-FOBT. The detection rate of CRC was $1.05 \%$ and the positivity rate of the method 
Table 1 i-FOBT-test performance compared with colonoscopy

\begin{tabular}{lccr}
\hline Predictive values & Any neoplastic lesions & Cancer & Adenoma \\
\hline Sensitivity (\%) & $31.9(24.1-40.4)$ & $91.7(61.5-99.8)$ & $26.7(19.0-35.5)$ \\
Specificity (\%) & $83.4(77.2-88.5)$ & $79.6(74.6-84.0)$ & $83.7(77.6-88.7)$ \\
Positive predictive value (\%) & $58.9(46.8-70.3)$ & $15.1(7.8-25.4)$ & $51.6(38.6-64.5)$ \\
Negative predictive value (\%) & $62.1(55.7-68.3)$ & $99.6(97.7-100)$ & $63.6(57.2-69.7)$ \\
Positive likelihood ratio & $1.92(1.28-2.89)$ & $4.49(3.40-5.95)$ & $1.64(1.05-2.54)$ \\
Negative likelihood ratio & $0.82(0.72-0.93)$ & $0.10(0.02-0.68)$ & $0.88(0.77-0.99)$ \\
Prevalence (\%) & $42.7(37.2-48.4)$ & $3.80(1.98-6.54)$ & $39.5(33.9-45.2)$ \\
\hline
\end{tabular}

Confidence Interval CI 95\%

i-FOBT, immunochemical fecal occult blood test

\section{Summary Box}

\section{What is already known:}

- Guaiac fecal occult blood test screening has been shown to decrease the incidence and mortality of colorectal cancer

- Although long-term, large, programmatic trials with immunochemical fecal occult blood testing (i-FOBT) have not yet been completed, prospective data support the effectiveness of i-FOBT as a screening tool, including some evidence that programmatic testing reduces colorectal cancer (CRC) mortality

- There are several varieties of the new immunochemical test with different sensitivities in different populations

\section{What the new findings are:}

- This prospective study of 1039 symptom-free participants showed a high compliance and high detection rates for cancers and high-risk adenomas

- The OC-Light test is a practical method for CRC detection in an average-risk population

- The OC-Light test has a very high sensitivity and negative predictive value

was $9.7 \%$, being similar to the findings of other studies that employed this technique $[3,4]$. Lesions in the lower gastrointestinal tract were more frequent among participants with a positive i-FOBT result than among those with a negative result. The OC-Light test is a practical method for CRC detection in an average-risk population, since it is an easy one-step procedure, with high sensitivity, no dietary restrictions, and the result can be ready within 5 min after the delivery of hygienic sampling bottles.

In summary, we suggest that the OC-light test could be offered to a population that refuses to be screened by colonoscopy, given its huge positive predictive value for CRC.
However, patients need to understand that the test has a low negative predictive value for any neoplastic lesion.

\section{References}

1. Souza DL, Jerez-Roig J, Cabral FJ, de Lima JR, Rutalira MK, Costa JA. Colorectal cancer mortality in Brazil: predictions until the year 2025 and cancer control implications. Dis Colon Rectum 2014;57:1082-1089.

2. Levi Z, Birkenfel S, Vilkin A, et al. A higher detection rate for colorectal cancer and advanced adenomatous polyp for screening with immunochemical fecal occult blood test than guaiac fecal occult blood test, despite lower compliance rate. A prospective, controlled, feasibility study. Int J Cancer 2011;10:2415-2424.

3. Castiglione G, Zappa M, Grazzini G, et al. Screening for colorectal cancer by fecal occult blood test: comparison of immunochemical tests. J Med Screen 2000;7:35-37.

4. Fenocchi E, Martínez L, Tolve J, et al. Screening for colorectal cancer in Uruguay with an immunochemical fecal occult blood test. Eur J Cancer Prev 2006;15:384-390.

5. Guittet L, Bouvier V, Mariotte N, et al. Comparison of a guaiac based and an immunochemical fecal occult blood test in screening for colorectal cancer in a general average risk population. Gut 2007;56:210-214.

6. Haug U, Kuntz KM, Knudsen AB, Hundt S, Brenner H. Sensitivity of immunochemical fecal occult blood testing for detecting left- vs right-sided colorectal neoplasia. Br J Cancer 2011;104:1779-1785.

7. Bini EJ. Use of upper endoscopy to evaluate patients with a positive fecal occult blood test and negative colonoscopy: is it appropriate? Dig Liver Dis 2006;38:507-510.

8. Rockey DC, Koch J, Cello JP, Sanders LL, McQuaid K. Relative frequency of upper gastrointestinal and colonic lesions in patients with positive fecal occult-blood tests. $N$ Engl J Med 1998;339:153-159.

9. Zorzi M, Fedeli U, Schievano E, et al. Impact on colorectal cancer mortality of screening programmes based on the fecal immunochemical test. Gut 2015;64:784-790.

10. Ventura L, Mantellini P, Grazzini G, et al. The impact of immunochemical fecal occult blood testing on colorectal cancer incidence. Dig Liver Dis 2014;46:82-86.

11. McClements PL, Madurasinghe V, Thomson CS, et al. Impact of the UK colorectal cancer screening pilot studies on incidence, stage distribution and mortality trends. Cancer Epidemiol 2012;36:e232-e242.

12. Tsuji Y, Gunji T, Sato H, et al. Antithrombotic drug does not affect the positive predictive value of an immunochemical fecal occult blood test. Dig Endosc 2014;26:424-429. 